\title{
A Case of Delayed Diagnostic Pulmonary Tuberculosis during Targeted Therapy in an EGFR Mutant Non-Small Cell Lung Cancer Patient
}

\author{
Caibao Jin Bin Yang \\ Department of Thoracic Oncology, Hubei Cancer Hospital, Wuhan, China
}

\author{
Keywords \\ Lung cancer · Pulmonary tuberculosis · EGFR
}

\begin{abstract}
The coexistence of lung cancer and pulmonary tuberculosis (TB) is rare, and the clinical and radiological features are always similar between lung cancer and pulmonary TB. In the present case, a non-small cell lung cancer patient with an epidermal growth factor receptor (EGFR)sensitive mutation was diagnosed with pulmonary TB during the treatment of tyrosine kinase inhibitor (TKI) because of the discrepant and confusing responses among different lesions. Therefore, we should combine clinical and radiological characteristics with pathological and microbiological tests to confirm the diagnosis of TB or lung cancer. It is a safe and selectable therapeutic strategy to treat EGFR mutant lung cancer patients with active TB using anti-TB medications and TKIs simultaneously.

\section{Introduction}

Lung cancer is the leading cause of cancer-related deaths, while tuberculosis (TB) is the main cause of infection-related deaths in the world. It has been reported that TB may increase the risk of lung cancer and influence the prognosis of lung cancer. A population-based casecontrol study found that pulmonary TB was significantly associated with lung cancer after adjustment for active smoking and socioeconomic status, with an odds ratio of 2.1 [1]. A nationwide cohort study in Korea found that the hazard ratio of lung cancer with old pulmonary TB was 3.24 compared to the control group [2]. Engels et al. [3] retrospectively analyzed 42,422 farmers in China, finding that lung cancer mortality was higher in subjects with TB (25 vs. 3.1 per 1,000 person years). 


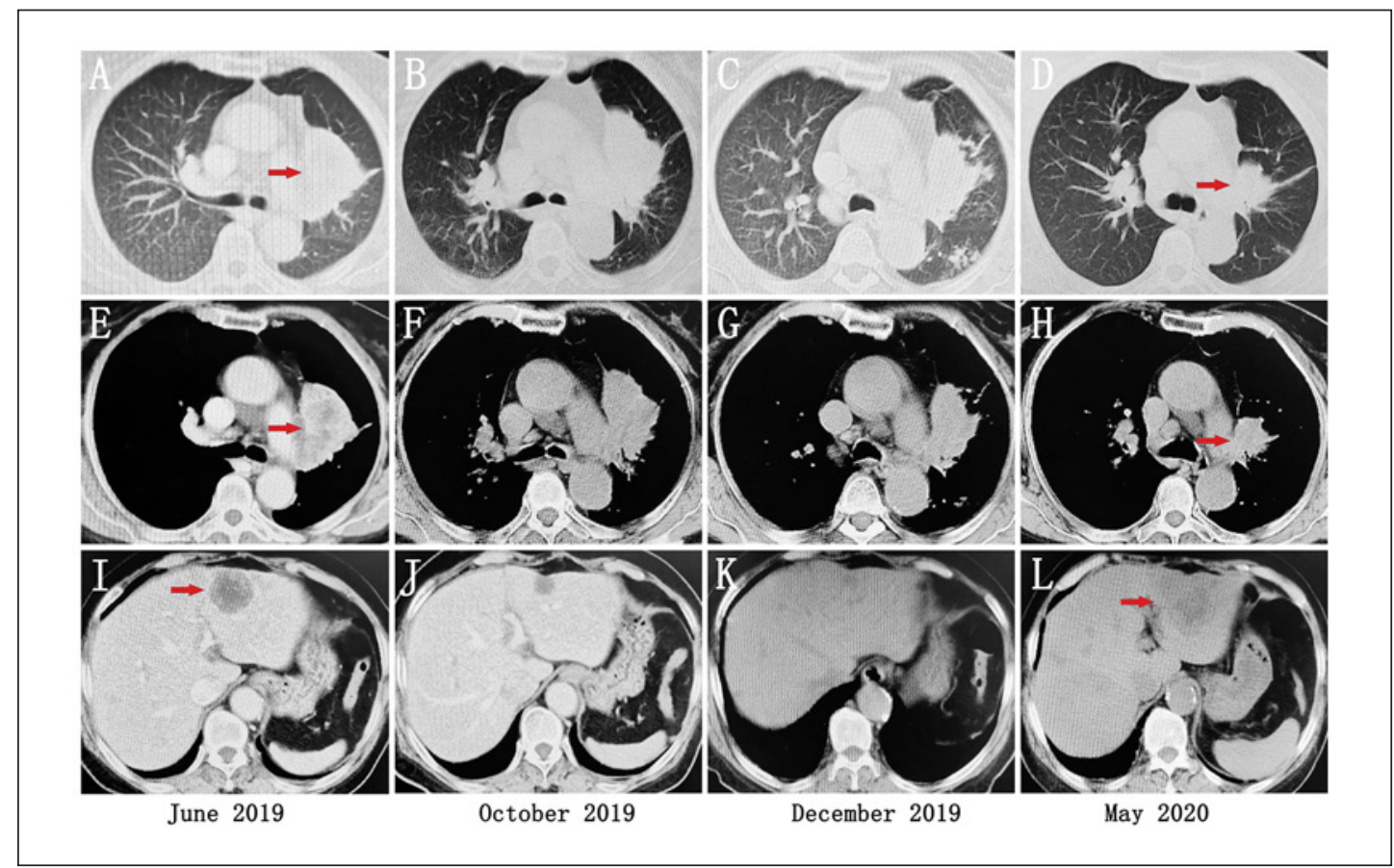

Fig. 1. Chest and abdomen CT was performed at diagnosis (A, E, I), after 6 months of treatment with icotinib $(\mathbf{B}, \mathbf{F}, \mathbf{J})$, after combination treatment of icotinib and chemotherapy $(\mathbf{C}, \mathbf{G}, \mathbf{K})$, and after anti-TB and targeted therapy (D, H, L).

The coexistence of pulmonary TB and lung cancer is rare, with an estimated incidence rate of approximately 2\% [4]. Varol et al. [5] retrospectively evaluated 3,350 active TB patients, $1.1 \%$ of which had coexisting lung cancer. Squamous cell lung cancer was the predominant histology. The clinical and radiological features of pulmonary TB are always similar to that of lung cancer, which could lead to a misdiagnosis, missed diagnosis, or delayed diagnosis. Herein, we report a case of delayed diagnostic pulmonary TB during the treatment of tyrosine kinase inhibitor (TKI) in epidermal growth factor receptor (EGFR) mutant nonsmall cell lung cancer (NSCLC).

\section{Case Report}

In June 2019, a 70-year-old woman was referred to our hospital due to pain in the chest and back. Chest computed tomography (CT) revealed a tumor in the upper left lobe involving the left hilum and mediastinum (Fig. 1A, E). Moreover, multiple metastatic sites were suspected at mediastinal lymph nodes, ribs, liver, and left adrenal gland (Fig. 1I). The pathological result of biopsy of liver metastases showed adenocarcinoma originating from the lung. After complete inspection, the patient was diagnosed as stage IV NSCLC (adenocarcinoma). The gene detection of biopsy tissue by amplification refractory mutation system (ARMS) showed deletion in exon19 at the EGFR gene. Rearrangement in the anaplastic lymphoma kinase (ALK) and c-Ros oncogene 1 receptor kinase (ROS1) fusions were negative. Afterwards, the patient started to receive targeted therapy of icotinib $(125 \mathrm{mg}$, per os, t.i.d.). Re-examination by radiological scans in 1-month intervals demonstrated the primary lung cancer mass had no obvious change but the remaining metastasis of the liver, adrenal gland, 
and mediastinal lymph nodes had decreased in size significantly (Fig. 1B, F, J). According to the Response Evaluation Criteria in Solid Tumors v.1.1 (RECIST 1.1), a stable response in pulmonary lesions and partial response in extrapulmonary metastasis were confirmed. In November 2019, the patient received two cycles of chemotherapy with pemetrexed on the basis of targeted therapy. However, the pulmonary tumor remained stable after combination therapy (Fig. 1C, G). In order to clarify the different responses between the primary pulmonary lesion and other metastasis, electronic bronchoscope was performed, which revealed an ulcer at the left main and superior lobar bronchus. Also, a biopsy specimen revealed chronic inflammation with necrosis. A nucleic acid test of respiratory pathogens in the sputum was positive for mycobacterium TB complex. The patient was then transferred to the TB hospital. GeneXpert MTB/RIF detection showed a positive result but a TB smear was negative. After the diagnosis of TB in January 2020, the patient received anti-TB treatment with an HRZE fixed dose combination composed of isoniazid, rifampicin, pyrazinamide, and ethambutol for 2 months. Meanwhile, she continued targeted therapy. In May 2020, chest and abdomen CT demonstrated that the pulmonary mass had shrunk significantly but metastases of the liver and adrenal gland had enlarged markedly, which was assessed as disease progression (Fig. 1D, $\mathrm{H}, \mathrm{L}$ ). Re-biopsy of the liver metastasis showed the same pathological pattern as before. The gene detection by ARMS revealed deletion in exon19 and a point mutation in exon 20 (T790M) at the EGFR gene. Hence, the patient received osimertinib treatment.

\section{Discussion}

Usually, we cannot distinguish between lung cancer and TB simply through radiological features and clinical symptoms, both of which are extremely similar. In a retrospective study to identify the imaging characteristics of asymptomatic pulmonary TB according to CT or ${ }^{18} \mathrm{~F}$-fludeoxyglucose positron emission tomography CT (PET-CT) presentations, pulmonary TB exhibited suspected malignant signs on CT imaging. Compared with lung cancer using PET-CT, the maximum standard uptake value (SUVmax) was lower in TB ( $p=0.036)$. The optimal cut-off level was SUVmax 8.45 with a sensitivity of $63 \%$ and specificity of $88.9 \%$ [6]. In the present case, the chest CT imaging revealed a malignant tumor. Because of the differing response of pulmonary mass and other metastases such as the liver and adrenal gland, further bronchoscopy and TB-related molecular tests were performed supporting the diagnosis of pulmonary TB. Furthermore, the pulmonary tumor obviously shrank after anti-TB therapy. Although the pathological result of bronchoscopic biopsy did not suggest a malignant tumor, we considered that TB coexisted with the primary site of lung cancer in the same neoplasm. Therefore, the integrated analysis of clinical and radiological features, pathological biopsy, etiological detection, and the response to anti-tumor or anti-TB therapy may be beneficial to differentiate TB with lung cancer.

The relationship between TB and lung cancer has been studied extensively. Most studies indicated an increased risk of lung cancer with a history of TB. A meta-analysis of 30 studies found that the relative risk of lung cancer for subjects with TB was 1.76 (95\% CI 1.49, 2.08) [7]. Yu et al. [8] prospectively analyzed an eligible, insured adult population of 716,872 in Taiwan, revealing an 11-fold higher incidence of lung cancer in patients with TB than non-TB subjects ( 26.3 vs. 2.41 per 10,000 person years). In our present case, the patient had lung cancer and pulmonary TB concurrently with no history of TB. There are some related theories about a causal relationship between lung cancer and TB. One theory supports that lung cancer could result from a previous TB lesion. The chronic inflammatory responses play a crucial role in cancer development and progression. Pulmonary TB could cause tissue damage persistently through the release of inflammatory factors such as tumor necrosis

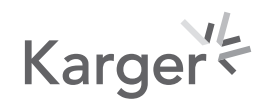


factor, interferon gamma, and interleukin [9]. In addition, the tissue repair process also participates in the development of lung cancer through the production of fibrosis and formation of new blood vessels. During the tissue damage and repair course, the lung epithelium DNA mutations could occur and ultimately lead to carcinogenesis. Another theory suggests that lung cancer could reactivate the tubercle bacilli in areas of inactive TB, or increase the susceptibility to opportunistic infections, especially in patients under antitumor treatment [10]. In our case, we could not determine the causal relationship between lung cancer and TB.

In the era of precision medicine, EGFR-TKIs have been the preferred standard therapeutic strategy for advanced or metastatic NSCLC with sensitive EGFR mutations. Hwang et al. [11] analyzed 477 patients with pulmonary adenocarcinoma to study the differences in EGFR mutational status, response to TKIs, and survival between patients with preexisting TB lesions (TB group) and those without the lesions (non-TB group). Compared with the non-TB group, the frequency of EGFR mutation was significantly higher in the TB group (56 vs. $34 \%, p=0.038$ ). Meanwhile, both the progression-free survival (PFS; 9.1 vs. 11.6 months, $p=0.02$ ) and overall survival (19.4 vs. 24.5 months, $p=0.014$ ) after first-line EGFR-TKI treatment were significantly shorter in the TB group. However, there were only limited case reports of active TB coexisting with EGFR mutant lung cancer $[12,13]$. The influence of active TB on the prognosis of coexisting lung cancer is unclear on the basis of current data. In our case, PFS with first-line treatment with icotinib was 10 months, during which the patient also received two cycles of chemotherapy. In a prospective randomized controlled trial [14], PFS in the combination group of icotinib plus chemotherapy was 16 months, which is longer than that of our case. It has been reported that coadministration of rifampicin decreases the plasma concentrations and efficacy of EGFR-TKIs. The patient in our case also received anti-TB therapy, including rifampicin for 2 months, which may have affected the efficacy.

\section{Conclusion}

It is crucial to combine clinical and radiological characteristics with pathological and microbiological tests to confirm the diagnosis of TB or lung cancer. We should not ignore the possibility of TB coexisting with lung cancer. Although anti-TB drugs like rifampicin may influence the efficacy of EGFR-TKIs, it is a safe and selectable therapeutic strategy to treat EGFR mutant lung cancer patients with active TB with anti-TB medications and TKIs simultaneously.

\section{Statement of Ethics}

The patient provided written informed consent for publication of this case report and any accompanying images.

\section{Conflict of Interest Statement}

The authors report no conflicts of interest in this work. 


\section{Funding Sources}

There were no funding sources.

\section{Author Contributions}

C.J. was responsible for writing the manuscript. B.Y. mainly participated in manuscript revision.

\section{References}

1 Brenner AV, Wang Z, Kleinerman RA, Wang L, Zhang S, Metayer C, et al. Previous pulmonary diseases and risk of lung cancer in Gansu Province, China. Int J Epidemiol. 2001;30(1):118-24.

2 Oh CM, Roh YH, Lim D, Kong HJ, Cho H, Hwangbo B, et al. Pulmonary tuberculosis is associated with elevated risk of lung cancer in Korea: the Nationwide Cohort Study. J Cancer. 2020;11(7):1899-906.

3 Engels EA, Shen M, Chapman RS, Pfeiffer RM, Yu YY, He X, et al. Tuberculosis and subsequent risk of lung cancer in Xuanwei, China. Int J Cancer. 2009;124(5):1183-7.

4 Byun HG, Yoo JY, Kim SJ, Lee OJ, Yoo MY. Coexistence of lung adenocarcinoma and pulmonary tuberculosis within a single lesion: a rare case report. Medicine. 2019;98(39):e17378.

5 Varol Y, Varol U, Unlu M, Kayaalp I, Ayranci A, Dereli MS, et al. Primary lung cancer coexisting with active pulmonary tuberculosis. Int J Tuberc Lung Dis. 2014;18(9):1121-5.

6 Lang S, Sun J, Wang X, Xiao Y, Wang J, Zhang M, et al. Asymptomatic pulmonary tuberculosis mimicking lung cancer on imaging: a retrospective study. Exp Ther Med. 2017;14(3):2180-8.

7 Brenner DR, McLaughlin JR, Hung RJ. Previous lung diseases and lung cancer risk: a systematic review and meta-analysis. PLoS One. 2011;6(3):e17479.

8 Yu YH, Liao CC, Hsu WH, Chen HJ, Liao WC, Muo CH, et al. Increased lung cancer risk among patients with pulmonary tuberculosis: a population cohort study. J Thorac Oncol. 2011;6(1):32-7.

9 Cooper AM, Khader SA. The role of cytokines in the initiation, expansion, and control of cellular immunity to tuberculosis. Immunol Rev. 2008;226(1):191-204.

10 Kim YI, Goo JM, Kim HY, Song JW, Im JG. Coexisting bronchogenic carcinoma and pulmonary tuberculosis in the same lobe: radiologic findings and clinical significance. Korean J Radiol. 2001;2(3):138-44.

11 Hwang IK, Paik SS, Lee SH. Impact of pulmonary tuberculosis on the EGFR mutational status and clinical outcome in patients with lung adenocarcinoma. Cancer Res Treat. 2019;51(1):158-68.

12 Lee HY, Kim JW, Yeo CD. A case of tuberculosis reactivation suspected of cancer progression during oral tyrosine kinase inhibitor treatment in a patient diagnosed as non-small cell lung cancer. J Thorac Dis. 2017; 9(8):E709-13.

13 Gothi D, Spalgais S. Tuberculosis in epidermal growth factor receptor mutation in lung adenocarcinoma on treatment with gefitinib/erlotinib. Indian J Cancer. 2017 Jan-Mar;54(1):1.

14 Xu L, Qi Q, Zhang Y, Cui J, Liu R, Li Y. Combination of icotinib and chemotherapy as first-line treatment for advanced lung adenocarcinoma in patients with sensitive EGFR mutations: a randomized controlled study. Lung Cancer. 2019;133:23-31.

\section{Karger'k}

